\title{
Anesthetic management for thoracoabdominal aneurysm repair performed under left heart bypass with renal and abdominal viscera selective perfusion
}

\author{
Vázquez Antas M., Santiago Paniagua P., Arcas Bellas J.J., Martín Piñeiro B., Stanciu O., Muñoz Alameda L.E. \\ Hospital Fundación Jimenez Díaz, Dept of Anaesthesiology \& Intensive Care, Madrid, Spain
}

\section{BACKGROUND:}

Open surgery for repair of thoracoabdominal aortic aneurysms is considered among the most complicated surgeries nowadays, with high mobility and mortality. Hemodynamic changes, one lung ventilation, adequate perfusion of abdominal viscera, as well as the risk of spinal cord ischemia and high transfusion rate, are among the possible difficulties.

\section{CASE REPORT:}

55-years-old woman, with hypertension, HIV and an incidental type II thoracoabdominal aortic aneurismal dilatation discovered during anaesthetic assessment for knee arthroplasty.

The surgery is planned to be performed under left heart bypass with renal and abdominal viscera selective perfusion.

\section{DISCUSSION:}

The procedure is performed under balanced general anesthesia with left-side double lumen endotracheal tube. Invasive monitoring (left radial artery, swan-ganz catheter and 4 lumen right jugular vein catheter) is used and a CSF drainage catheter is placed before surgery.

A left thoracotomy approach is performed. For the left heart bypass two cannulas are placed, the proximal one in the aortic arch distal to subclavian artery and the distal one in the left femoral artery. The prosthesis is placed between the aortic arch and the iliac bifurcation.

Postoperative evolution in ICU and ward was eventless and renal and neurological functions (both sensitive and motor) were preserved at all times. 60 days after hospital admission the patient was discharged from hospital.

Prevention of ischemia both at the abdominal and spinal levels is essential during the repair of type II thoracoabdominal aortic aneurysms. To achieve it, retrograde distal aortic perfusion was performed by the left heart bypass with selective renal and abdominal viscera perfusion; also CSF was drained to maintain pressures bellow $10 \mathrm{mmHg}$ during the entire procedure.
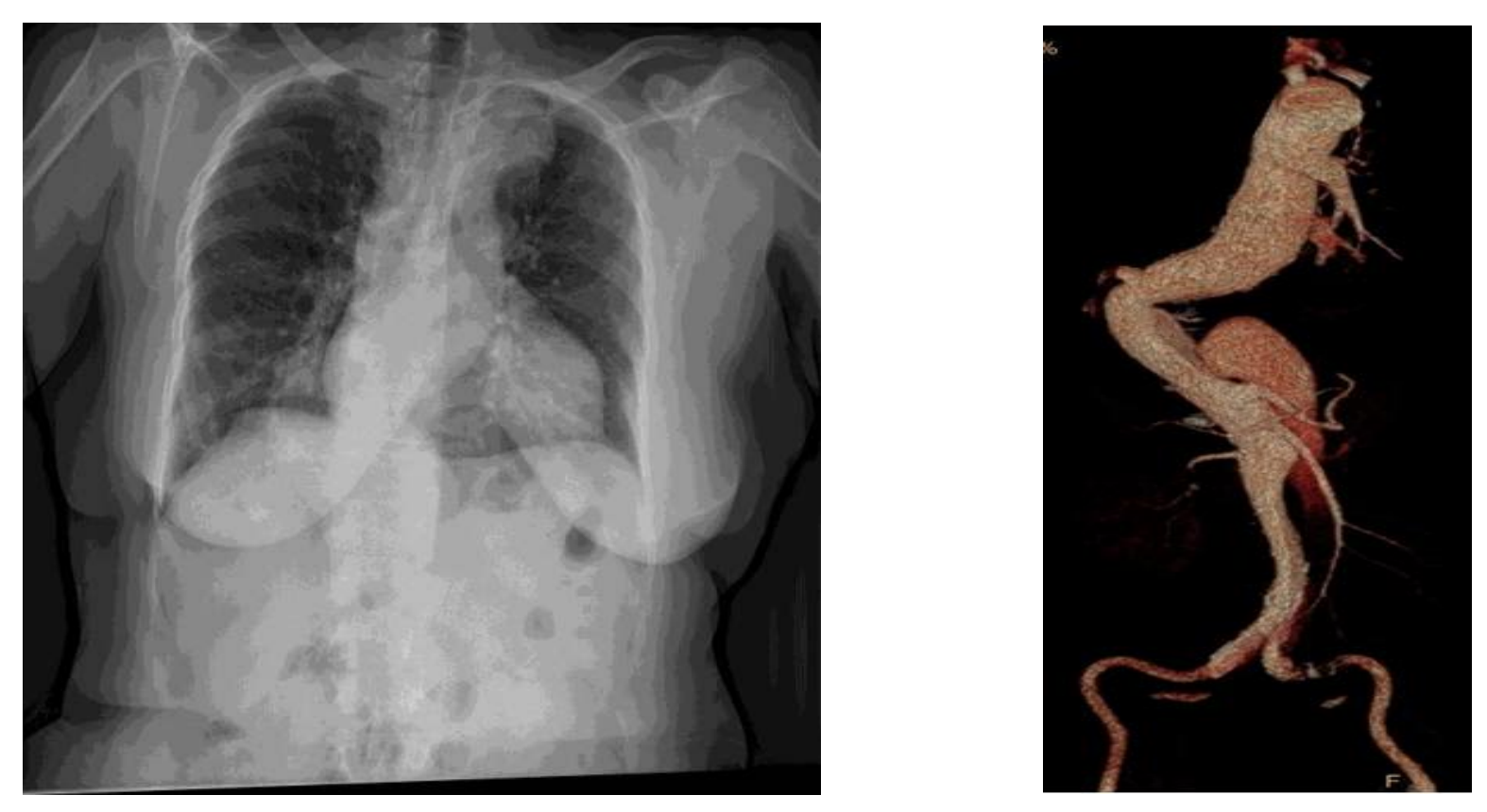

\section{LEARNING POINTS:}

Type II thoracoabdominal aortic aneurysms repair performed with left heart bypass allows to maintain perfusion of the lower half of the body through a retrograde distal aortic perfusion, decreasing ischemic injuries and improving prognosis.
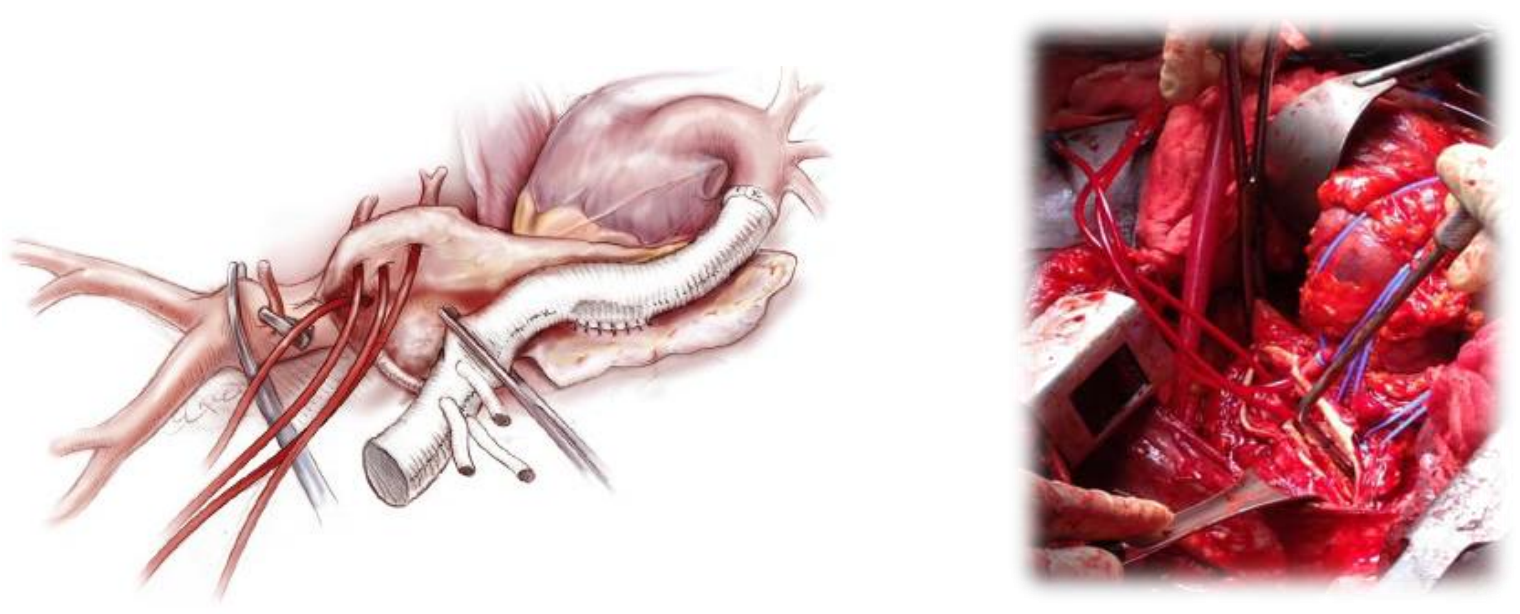